\title{
1 Protecting half the planet could directly affect over one billion people
}

2 Judith Schleicher ${ }^{\mathrm{a},{ }^{*}}$, Julie G. Zaehringer ${ }^{\mathrm{a}, \mathrm{b}}$, Constance Fastre ${ }^{\mathrm{c}}$, Bhaskar Vira ${ }^{\mathrm{a}}$, Piero Visconti ${ }^{\mathrm{d}}$ and

3 Chris Sandbrook ${ }^{a}$

$4 \quad{ }^{a}$ Department of Geography, University of Cambridge, Cambridge CB2 3EN, UK.

$5 \quad{ }^{\mathrm{b}}$ Centre for Development and Environment, University of Bern, Bern, Switzerland.

6 ' Institute of Zoology, Zoological Society of London, Regent's Park, London, NW1 4RY, UK.

7 d International Institute for Applied Systems Analysis (IIASA), Schlossplatz 1, 2361 Laxenburg, Austria.

$8 \quad{ }^{*}$ Corresponding author: Judith.Schleicher@cantab.net; 


\section{Abstract}

10 In light of continuing biodiversity loss globally, one ambitious proposal has gained 11 considerable traction amongst conservationists: the goal to protect half the Earth. Our 12 analysis suggests that at least 1 billion people live in places that would be protected if the 13 Half Earth proposal were implemented within all ecoregions. Considering the social and 14 economic impacts of such proposals is central to addressing social and environmental justice concerns, and assessing their acceptability and feasibility.

\section{Main text}

18 To halt the rapid loss of biodiversity globally, numerous conservation strategies have been 19 implemented. Member states of the Convention on Biological Diversity (CBD) have 20 committed to placing $17 \%$ and $10 \%$ of the world's terrestrial and marine areas, respectively, 21 within protected areas (PAs) by 2020 (Aichi Biodiversity Target $11^{1}$ ). Although meeting this 22 target is within reach in many countries ${ }^{2}$, rapid biodiversity loss continues ${ }^{3}$. As a result, 23 conservationists have responded with alternative and more ambitious goals. One prominent 24 proposal calls for the expansion of the global conservation estate to cover half the Earth ${ }^{4,5}$.

25 This Half Earth, or Nature Needs Half, proposal has gained strong momentum, and has the potential to influence the post-2020 biodiversity targets and related processes ${ }^{6}$. Indeed, the Global Deal for Nature (GDN), which aims for 30\% protection by 2030 and $50 \%$ by 2050, has been endorsed by a broad coalition of environmental organisations ${ }^{7}$. across the planet. So far, the proposal has received some scrutiny with regards to

31 environmental considerations ${ }^{8}$ and its potential impacts on food production ${ }^{9}$. However, there

32 has been no empirical analysis of other social and economic impacts of Half Earth, and the 
proposal itself has been ambiguous about the exact forms and location of new conserved areas being called for. This is despite the fact that the proposal's social and economic 35 impacts will influence its ability to deliver its conservation objectives and that there are frequently trade-offs involved in meeting environmental, social and economic goals of 37 conservation and development interventions ${ }^{10,11}$. The reported impacts of existing PAs vary widely from physical and economic displacement to positive socio-economic outcomes for well-being or industry ${ }^{12}$. These impacts depend in part on the type of PAs, their governance arrangements, and the restrictions they place on resource use. Where the impacts are negative, they tend to disproportionally affect marginalised communities ${ }^{13}$. In light of this evidence on existing PAs, the increase in conserved areas to $50 \%$ could have large implications for the lives of those living inside these areas or in their vicinity ${ }^{14,15}$.

We investigated the human implications of Half Earth by assessing the number and 45 distribution of people that would be directly affected if half of Earth's land mass was protected. Since there is no consensus among those calling for a $50 \%$ protection target regarding which additional areas to protect, we based our analysis on the ecoregion approach proposed by Dinerstein and colleagues $^{78}$. This approach is based on 846 ecoregions, to ensure protection of the full range of ecosystems and their associated species,

50 to adequately conserve all elements of biodiversity. Dinerstein et al. ${ }^{8}$ classify the ecoregions

51 into four categories: those that already have $50 \%$ protection, those that could achieve $50 \%$

52 protection as sufficient natural habitat remains, those where $50 \%$ could be possible with 53 substantial restoration efforts, and those with at most $20 \%$ of their natural habitat remaining 54 and where achieving $50 \%$ protection of habitat is therefore unrealistic. To calculate the 55 minimum number of people who would live in the conserved areas, and hence, would be 56 directly affected by Half Earth, we selected areas ( $5 \times 5 \mathrm{~km}$ pixels) to be added to the existing 
57 PA network within each ecoregion from lowest to highest human footprint value ${ }^{16}$ until $50 \%$

58

coverage was achieved under two scenarios: (a) within all ecoregions and (b) only in ecoregions where Dinerstein and colleagues consider Half Earth reachable ${ }^{8}$. To achieve this we combined the global data layers of ecoregions, PAs (from the World Database of Protected Areas $^{17}$ ) and human footprint with a global human population layer for $2017^{18}$.

Our approach assumes a protection strategy designed to minimise key impacts on society, including avoiding areas with high population density and agricultural land. It ignores effects of conserved areas on people living beyond their boundaries, such as constrained access to resources. For these reasons our approach generates a conservative (lower bound) estimate of the potential number of people affected. Indeed, areas with higher human footprint values, and higher population density, would have to be protected if additional ecological criteria were applied to design the protection strategy, such as ensuring connectivity between conserved areas, setting minimum size thresholds of conserved areas, or seeking to protect land with highest biodiversity regardless of ecoregion. Hence, the number of people affected would likely be higher, especially in poorer countries which tend to have higher concentrations of biodiversity ${ }^{19}$.

We find that over 1 billion people currently live in areas that would be protected under Half Earth if the proposal were applied to all ecoregions (Fig. 1). This is four times the number of people estimated by our approach to be living in PAs today ( 247 million) and includes 760 million people living in additional areas to be protected beyond existing PAs to meet the $50 \%$ target. If we only consider the ecoregions where Dinerstein et al. suggest $50 \%$ protection is feasible ${ }^{8}, 28 \%$ of the ecoregions' area (Supplementary Figure 2), currently home to 170 million people, must be newly protected. This is roughly equivalent to the population of the UK, Thailand and Morocco combined. The majority of people living in new areas to be 
81 protected live in middle-income countries and $~ 10 \%$ in low income countries, regardless of

82 whether we include all, or only less impacted, ecoregions (Table 1).

83 The majority of the additional conserved areas have human footprint values within

84 the lowest 20\% (Supplementary Figure 3). However, the global network of conserved areas

85 necessary to achieve Half Earth would comprise areas with human footprint values within the 86 top $20 \%$ under both scenarios, covering all ecoregions or only less impacted ones. At the

87 upper end of this spectrum, these include highly developed areas, such as London, UK (Fig. 1

88 and Supplementary Figure 2). Implementing Half Earth at the ecoregion level in this way

89 would clearly be in conflict with human use, raising questions about the feasibility and 90 diverse social implications of this strategy.

91 We recognise the importance of conserved areas for the future of life on Earth, and

92 the fundamental need for radical action in the face of unfolding environmental crises.

93 However, our findings highlight the crucial importance of taking into account the human

94 impacts of Half Earth, GDN, or other ambitious (area-based) conservation targets. Even with

95 our conservative approach a very large number of people would be affected by

96 implementing Half Earth. Therefore, any such proposals need to explicitly consider and

97 seriously engage with their social and economic consequences. Considering these

98 implications is not only central to concerns about social and environmental justice, but will

99 also determine how realistic their implementation is in terms of achieving their intended

100 conservation outcomes.

101 Based on our findings we make three recommendations. Firstly, Half Earth 102 proponents should be explicit about the types, and location, of conserved areas they are 103 calling for, to allow for more in-depth assessments of their social, economic and 104 environmental impacts in the future. Secondly, the advocates of all area-based conservation 
measures should recognise and take seriously the human consequences, both negative and positive, of their proposals. Thirdly, the Parties to the CBD, tasked with negotiating and implementing the post-2020 conservation framework, should apply more holistic, interdisciplinary approaches that take into account social and economic implications across scales $^{14,20}$. Such approaches should consider important broader issues such as environmental justice, the plural values people attribute to nature, and the need for action to tackle the ultimate economic consumption and production drivers of biodiversity loss ${ }^{10,14,21}$.

\section{Methods}

To determine the number and distribution of people living in areas that would be protected under two Half Earth scenarios (50\% protection within all ecoregions, and $50 \%$ protection of those ecoregions with more than $20 \%$ natural habitat remaining), we combined the following global datasets: terrestrial ecoregions ${ }^{8}$, human footprint ${ }^{16}$, the World Database of PAs (WDPA, version July $2018^{17}$ ) and LandScan 2017 global population distribution ${ }^{18}$. We focused on ecoregions because (a) Half Earth targets have been judged achievable, or already reached, in $\sim 49 \%$ of all ecoregions ${ }^{8}$, (b) they have been widely used as a proxy to capture biodiversity for conservation planning, and (c) they are the basis for the GDN proposal ${ }^{7}$ and for assessing Half Earth's impacts on food production? ${ }^{9}$. We grouped ecoregions into Dinerstein et al.'s $\mathrm{s}^{8}$ four categories according to their percentage protection and the amount of natural habitat remaining. We selected new areas for protection (here referred to as 'conserved areas') based on the human footprint, which combines a diversity of human impacts, including human population density, agricultural land, infrastructure and transport routes. While it does not capture some less intensive human influences, it is the most comprehensive global index of its kind. To determine the distribution of people within 
countries of different income-status, we joined a Global Administrative Areas (GADM) layer at country level ${ }^{22}$ with the World Bank's (WB) income classification ${ }^{23}$ of low, low-middle, upper-middle and high income countries. Disputed territories and countries without WB income codes were excluded from the analysis $(n=6)$.

We pre-processed datasets in ArcGIS version 10.4.1. We rasterized all datasets, projected them to Mollweide equal area at a spatial resolution of $\sim 5 \times 5 \mathrm{~km}$, and set them to a common extent. Through this pre-processing very small ecoregions, covering less than $50 \%$ of any pixel, were removed, resulting in 818 remaining ecoregions. We excluded Antarctica because it is not included in the human footprint dataset nor in the analysis conducted by Dinerstein and colleagues ${ }^{8}$. As Antarctica is not permanently settled, excluding it does not affect our population count results.

We imported, stacked and analysed the raster datasets in $\mathrm{R}$ version $3.5 .1^{24}$. To determine the area to be protected in each ecoregion to meet the $50 \%$ target, we divided the total area of each ecoregion by two and subtracted the area currently protected per ecoregion according to WDPA ${ }^{17}$. Under the first scenario, we then ordered pixels in each ecoregion according to ascending human footprint values and selected the number of pixels with the lowest human footprint values to meet the $50 \%$ target within each ecoregion from pixels not under protection. We calculated the number of people living in the selected areas by summing up the population count value ${ }^{18}$. Additionally, we calculated the number of people living within existing PAs by combining the WDPA with the population distribution data layer. Under the second scenario, we repeated this analysis while only selecting pixels to be protected from ecoregions where over $20 \%$ of natural habitat remains. Finally, we calculated the number of people living inside the conserved areas under each of these two scenarios per country, according to the WB income classification ${ }^{23}$. 
153

154

155

156

157

158

159

160

161

162

163

164

165

Data availability. The $\mathrm{R}$ Code to reproduce the results is provided in the Supplementary Information. The datasets used in this study are all publically available or available to educational institutions for non-commercial purposes, but not distributable by the authors. Details of each dataset and download links are provided in the Supplementary Information.

\section{References}

1. The Strategic Plan for Biodiversity 2011-2020 and the Aichi Biodiversity Targets (CBD, 2010).

2. Tittensor, D.P. et al. Science 346, 241-245 (2014).

3. Global Assessment Preview (IPBES, 2019).

4. Locke, H. George Wright Forum 31, 359-371 (2014).

5. Wilson, E.O. (Liveright, New York, 2016).

6. Dinerstein, E. et al. Sci. Adv. 5, eaaw2869 (2019).

7. Synthesis of Views of Parties and Observers on the Scope and Content of the Post-2020 Global Biodiversity Framework (CBD, 2019).

8. Dinerstein, E. et al. Bioscience 67, 534-545 (2017).

9. Mehrabi, Z., Ellis, E.C. \& Ramankutty, N. Nat. Sustain. 1, 409-412 (2018).

10. Ellis, E.C., Pascual, U. \& Mertz, O. Curr. Opin. Environ. Sustain. 38, 86-94 (2019).

11. Brockington, D. \& Wilkie, D. Philos. Trans. R. Soc. B Biol. Sci. 370, 20140271 (2015).

12. Oldekop, J.A., Holmes, G., Harris, W.E. \& Evans, K.L. Conserv. Biol. 30, 133-141 (2016).

13. West, P., Igoe, J. \& Brockington, D. Annu. Rev. Anthropol. 35, 251-277 (2006).

14. Büscher, B. et al. Oryx 51, 407-410 (2017).

15. Kopnina, H. Biol. Conserv. 203, 176-185 (2016).

16. Venter, O. et al. Nat. Commun. 7, 12558 (2016). 
17. World Database on Protected Areas (UNEP-WCMC \& IUCN, 2018). www.protectedplanet.net

18. Rose, A.N., McKee, J.J., Urban, M.L. \& Bright, E.A. Landscan 2017 (2018).

19. Balmford, A. et al. Science 291, 2616-2619 (2001).

20. Visconti, P., Bakkenes, M., Smith, R.J., Joppa, L. \& Sykes, R.E. Philos. Trans. R. Soc. B Biol. Sci. 370, 20140284 (2015).

21. Ten Brink, B. et al. (Netherlands Environmental Assessment Agency, 2010).

22. Database of Global Administrative Areas (GADM, 2018). www.gadm.org

23. Country Classification (World Bank, 2018).

24. R Core Team R: A language and environment for statistical computing (2018).

25. Google Earth (2019).

Corresponding Author. Correspondence to Judith Schleicher.

\section{Acknowledgements}

J.G.Z. undertook this work whilst a Visiting Scholar in the Department of Geography, University of Cambridge (May 2018-April 2019), and was supported by the Swiss Programme for Research on Global Issues for Development (r4d programme), which is funded by the Swiss National Science Foundation (SNSF) and the Swiss Agency for Development and Cooperation (SDC), under grant number 400440 152167. This product was made utilizing the LandScan (2017) ${ }^{\mathrm{TM}}$ High Resolution global Population Data Set copyrighted by UT-Battelle, LLC, operator of Oak Ridge National Laboratory under Contract No. DE-AC05-00OR22725 with the United States Department of Energy. 
J.S., J.G.Z., C.F., B.V., P.V., and C.S. designed the analyses. J.S. and J.G.Z. compiled the data and conducted the analyses. J.S. wrote the paper with input from J.G.Z., C.F., B.V., P.V., and

203 C.S.

Competing interests. The authors declare no competing interests.

Supplementary Information. The Supplementary Information contains Supplementary Notes, Methods and Figures, including the full R Code to reproduce the results.

\section{Figure captions:}

210 Table 1: Number of people (million) living in additional areas protected to meet Half Earth targets within each ecoregion, according to the World Bank classification of low, lower-

212 middle, upper-middle and high income countries and according to whether (a) all ecoregions 213 are included, or (b) only less impacted ecoregions, where more than $20 \%$ of natural habitat 214 remains. Percentage values of the total population are given for these two scenarios.

Fig. 1: Additional areas to be protected to meet Half Earth $50 \%$ protection targets within 217 each ecoregion, on a colour scale of increasing human footprint value. A to D illustrate additional conserved areas $(\sim 5 \times 5 \mathrm{~km})$ with the highest human footprint within each World Bank income class: (A) High: London, UK; (B) Upper-middle: St Lucia; (C) Lower-middle:

220 Egypt; (D) Low: Nepal ${ }^{25}$. 
Table 1: Number of people (million) living in additional areas protected to meet Half Earth targets within each ecoregion, according to the World Bank classification of low, lowermiddle, upper-middle and high income countries and according to whether (a) all ecoregions are included, or (b) only less impacted ecoregions, where more than $20 \%$ of natural habitat remains. Percentage values of the total population are given for these two scenarios.

\begin{tabular}{|l|c|c|}
\hline & All ecoregions & Less impacted ecoregions \\
\hline Low & $75(10 \%)$ & $16(9 \%)$ \\
\hline Lower-middle & $403(53 \%)$ & $64(37 \%)$ \\
\hline Upper-middle & $234(31 \%)$ & $65(38 \%)$ \\
\hline High & $47(6 \%)$ & $25(15 \%)$ \\
\hline
\end{tabular}



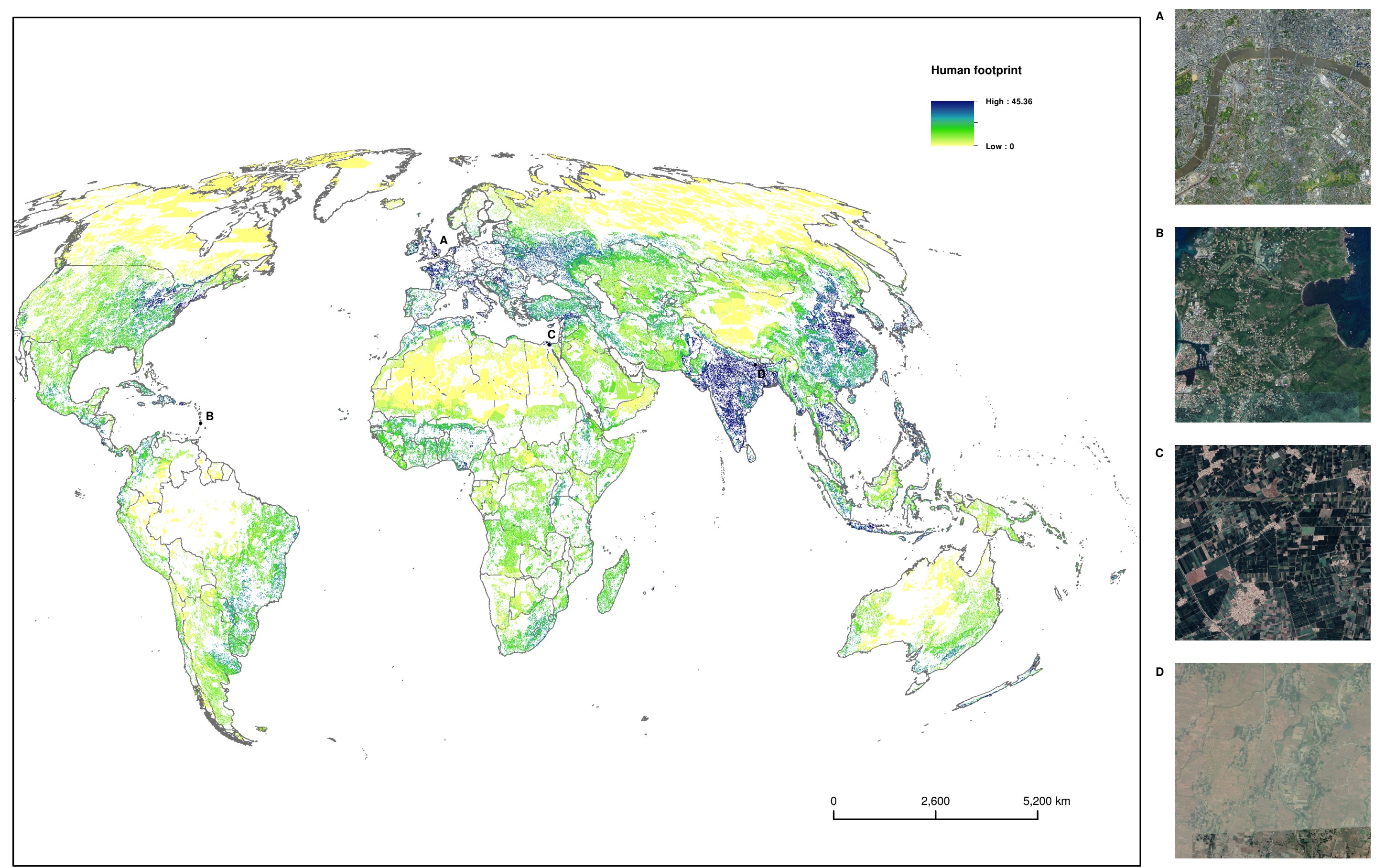